\title{
Mutant prevention concentration of orbifloxacin: comparison between Escherichia coli, Pseudomonas aeruginosa, and Staphylococcus pseudintermedius of canine origin
}

Takae Shimizu', Kazuki Harada ${ }^{1,2^{*}}$ and Yasushi Kataoka ${ }^{1}$

\begin{abstract}
Background: The mutant prevention concentration (MPC) is an important parameter to evaluate the likelihood of growth of fluoroquinolone-resistant mutants for antimicrobial-pathogen combinations. The MPCs of fluoroquinolones for different canine pathogens have not been compared. In this study, we compared for the first time orbifloxacin MPCs between susceptible strains of Escherichia coli, Pseudomonas aeruginosa, and Staphylococcus pseudintermedius of canine origin.
\end{abstract}

Methods: More than $10^{10} \mathrm{CFU} / \mathrm{ml}$ of 10 strains of each bacterial species were inoculated onto Muller-Hinton agar supplemented with different concentrations of orbifloxacin from $1 \times$ to $64 \times$ minimum inhibitory concentration (MIC) and the MPCs were recorded. MICs of original strains and of mutants arising after exposure to sub-MPC concentrations (one per original strain) were determined in the presence or absence of efflux pump inhibitors (EPIs). The effects of quinolone resistance-determining region (QRDR) mutations were also examined.

Results: MPCs were significantly higher for P. aeruginosa $(16-128 \mu \mathrm{g} / \mathrm{ml})$ than for E. coli $(0.5-32 \mu \mathrm{g} / \mathrm{ml})$. MPCs for S. pseudintermedius varied between the low-susceptible $(16-128 \mu \mathrm{g} / \mathrm{ml})$ and the high-susceptible strains $(4-16 \mu \mathrm{g} / \mathrm{ml})$ and were the most broadly distributed among the three species. Regarding resistance mechanisms, only one QRDR mutation in gyrA was found in all of the 10 mutants of $E$. coli and in 4 of the 10 mutants of $P$. aeruginosa, whereas mutations in both grlA and gyrA were found in 3 mutants and one mutation in grlA was found in 2 mutants among the 10 mutants of S. pseudintermedius. In the presence of an EPI, the MICs of P. aeruginosa mutants decreased markedly, those of E. coli mutants decreased moderately, and those of $S$. pseudintermedius mutants were unaffected.

Conclusions: MPCs of orbifloxacin vary between bacterial species of canine pathogens, possibly due to the diversity of the main fluoroquinolone resistance mechanism among these species. Therefore, the type of bacterial species should be taken into consideration when using fluoroquinolone drugs such as orbifloxacin in canines.

Keywords: Mutant prevention concentration, Fluoroquinolone, Escherichia coli, Pseudomonas aeruginosa, Staphylococcus pseudintermedius, Canines

\footnotetext{
* Correspondence: k-harada@muses.tottori-u.ac.jp

'Laboratory of Veterinary Microbiology, Nippon Veterinary and Life Science

University, 1-7-1, Kyonan-cho, Musashino, Tokyo 180-8602, Japan

${ }^{2}$ Department of Veterinary Internal Medicine, Tottori University, Minami

4-101, Koyama-Cho, Tottori-Shi, Tottori 680-8553, Japan
} 


\section{Background}

The incidence of canine pathogens showing decreased susceptibility or increased resistance to fluoroquinolones was reported in Japan [1-3] as well as worldwide. Determination of the mutant prevention concentration (MPC), the antimicrobial concentration that prevents selection of resistant mutants, is important for reduction of the incidence of fluoroquinolone resistance [4]. It is hypothesised that drug exposure below the MPC may promote selection of resistant strains and the concentration range between the minimum inhibitory concentration (MIC) and MPC, which is referred to as the mutant selection window (MSW), may enrich and amplify resistant mutants. Thus, MPC and MSW are important parameters for evaluation of the potential for emergence of fluoroquinolone-resistant mutants for antimicrobial-pathogen combinations [4]. MPCs of fluoroquinolone in canines have been sporadically reported for single pathogens [5-7]. However, a consistent comparison of fluoroquinolone MPCs among different pathogens has not been performed.

Resistance to fluoroquinolone is mediated primarily through mutations in the quinolone resistance-determining region (QRDR) of DNA gyrase and topoisomerase IV [8]. Decreased drug uptake due to overexpression of drug efflux pumps also contributes to development of fluoroquinolone resistance [8].

Orbifloxacin is a fluoroquinolone that was developed for use in veterinary medicine and has achieved a relatively large sales volume (approximately $137 \mathrm{~kg}$ in 2011) among fluoroquinolones for companion animals in Japan [9]. This antimicrobial agent exhibits bactericidal activity against numerous Gram-negative and Gram-positive bacteria (e.g. Escherichia coli, Pseudomonas aeruginosa, and Staphylococcus pseudintermedius) and is thus indicated for treatment of canine bacterial infections, including urinary, skin, and ear infections. Gebru et al. [6,7] found that orbifloxacin MPCs were relatively high compared to those of other veterinary fluoroquinolones, which may be helpful in establishing a comprehensive understanding of the variation of MPCs among different pathogens.

The purpose of the present study is to investigate differences in the likelihood of emergence of fluoroquinoloneresistant mutants among major bacterial pathogens based on MPC determination and to characterize the resistance mechanism of mutants. We compared the MPCs of orbifloxacin among fluoroquinolone-susceptible E. coli, P. aeruginosa, and S. pseudintermedius strains. Mutants arising after exposure to sub-MPC concentrations were screened for QRDR mutations and the effects of efflux pump inhibitors (EPIs) on the MICs of orbifloxacin were determined.

\section{Methods}

\section{Bacterial isolates}

Ten fluoroquinolone-susceptible strains each of the following three bacterial species were used in this study:
E. coli (strains E1-E10), P. aeruginosa (strains P1-P10), and S. pseudintermedius (strains S1-S10). E. coli and $P$. aeruginosa strains were selected from our collection of urine and ear/skin samples, respectively, obtained from domestic dogs [1,2]. S. pseudintermedius strains were isolated from swabs obtained from dogs with canine pyoderma at the Veterinary Medical Teaching Hospital, Nippon Veterinary and Life Sciences University, and at three veterinary hospitals located in Tokyo, Japan. Swabs were streaked onto mannitol salt agar (Eiken Chemical, Japan) and typical colonies were collected. Bacterial identification was carried out by Gram staining, catalase and coagulase tests, and multiplex-polymerase chain reaction (PCR) [10]. All confirmed S. pseudintermedius isolates were stored at $-80^{\circ} \mathrm{C}$ in $10 \%$ skimmed milk.

\section{Determination of MPCs and mutant recovery}

MPCs were determined using a previously described protocol [11] with slight modifications. A concentrated cellular suspension of each bacterial strain $(200 \mu \mathrm{l})$ containing $>10^{10}$ colony-forming units $(\mathrm{CFU}) / \mathrm{ml}$ was plated onto each of three Mueller-Hinton agar (Becton Dickinson, France) plates, which were supplemented with orbifloxacin at a concentration equal to the MIC and six doubling dilutions higher than the MIC (i.e. $2 \times$, $4 \times, 8 \times, 16 \times, 32 \times$, and $64 \times$ MIC). Plates were incubated at $37^{\circ} \mathrm{C}$ for 5 days because preliminary tests showed no significant differences in MPCs between incubations for 2 and 5 days, similarly with the previous report [11]. The lowest drug concentration that prevented the emergence of mutants after the 5-day incubation period was recorded as the MPC. Each experiment was performed twice.

A mutant of each original strain (EM1-EM10, PM1PM10, and SM1-SM10) was randomly selected from plates with a concentration of orbifloxacin that was one dilution (i.e. twofold) lower than the MPC (sub-MPC). Each mutant was cultured on antimicrobial-free agar plates for three serial passages and then stored at $-80^{\circ} \mathrm{C}$ until further analysis.

\section{Susceptibility testing for orbifloxacin}

MICs of orbifloxacin against the original strains and mutants were determined using the agar dilution method, according to the guidelines of the Clinical and Laboratory Standards Institute (CLSI) [12]. MICs of orbifloxacin were also determined in the presence of EPIs: $80 \mu \mathrm{g} / \mathrm{ml}$ of Phe-Arg- $\beta$-naphthylamide (PA $\beta \mathrm{N}$, Sigma-Aldrich, $\mathrm{MO}$, USA) for E. coli and P. aeruginosa, and $20 \mu \mathrm{g} / \mathrm{ml}$ reserpine (Sigma-Aldrich) for $S$. pseudintermedius. All inoculated agar plates were incubated at $35^{\circ} \mathrm{C}$ for $16-20$ h. E. coli ATCC 25922, P. aeruginosa ATCC27853, S. aureus ATCC29213, and Enterococcus faecalis ATCC29212 were used as quality control strains. 


\section{PCR amplification and DNA sequencing of QRDRs}

The QRDRs of the gyrA and parC genes for E. coli and $P$. aeruginosa or of the grlA and gyrA genes for $S$. pseudintermedius in the original strains and in representative mutants of each original strain were amplified by PCR using previously described primers [13-15]. The amplicons were bidirectionally sequenced using the PCR primers.

\section{Statistical analysis}

One-way analysis of variance (ANOVA) was used to compare MPCs and MPC/MIC, serum maximum concentration $\left(\mathrm{C}_{\text {max }}\right) / \mathrm{MPC}$, and area under the concentration time-curve (AUC)/MPC ratios among the three bacterial species, based on the results for ten original isolates per species. A Tukey test was used to evaluate differences among the geometric means of these parameters. A Welch test was used for pairwise comparison of MICs. The threshold for significance was set at a value of $P<0.05$ in all analyses.

\section{Results}

MICs of original strains and mutants in the presence or absence of EPIs

The results of the study are summarised in Table 1 . The MICs of orbifloxacin against the original strains and mutants were $0.063-2 \mu \mathrm{g} / \mathrm{ml}$ and $1-8 \mu \mathrm{g} / \mathrm{ml}$, respectively, for $E$. coli, and $1-4 \mu \mathrm{g} / \mathrm{ml}$ and $16-128 \mu \mathrm{g} / \mathrm{ml}$, respectively, for $P$. aeruginosa. Thus, the orbifloxacin MICs against the original strains of $P$. aeruginosa were significantly increased by drug exposure compared with those of the E. coli original strains (4- to 32 -fold vs. 2 - to 16 -fold, $P<0.05$ ).

For $S$. pseudintermedius, the MICs of orbifloxacin against the original strains were $0.25-1 \mu \mathrm{g} / \mathrm{ml}$. After drug exposure, the MICs of the high-susceptible strains (S1-S6) increased 1- to 4-fold, whereas those of the lowsusceptible strains (S7-S10) increased 8- to 64-fold. The MICs of orbifloxacin against the mutants of this species were widely distributed $(0.5-64 \mu \mathrm{g} / \mathrm{ml})$.

Addition of PA $\beta N$, an EPI, resulted in a decrease in the MICs of orbifloxacin against the mutants and original strains of $E$. coli and $P$. aeruginosa $(P<0.05)$ by 8 to 32 -fold and 32- to 512-fold, respectively. In contrast, the MICs of orbifloxacin against the original strains and mutants of $S$. pseudintermedius were unaffected by addition of reserpine $(P>0.05)$. By comparison of the MICs of mutants for all three bacterial species, the decrease in the MIC of orbifloxacin against $P$. aeruginosa was more pronounced, compared with those for $E$. coli and S. pseudintermedius $(P<0.05)$.

\section{QRDR mutations in original strains and mutants}

Sequence analysis of QRDRs revealed that four lowsusceptible original strains (E7-E10; MIC: $0.5-2 \mu \mathrm{g} / \mathrm{ml}$ ) and all the mutants of $E$. coli harboured one point mutation (Ser-83 $\rightarrow$ Leu or Asp-87 $\rightarrow$ Asn) in gyrA. In P. aeruginosa, four strains (PM4, PM7-PM9) harboured one point mutation $(\mathrm{Thr}-83 \rightarrow$ Ile). No mutations were found in the parC gene of $E$. coli or P. aeruginosa.

In S. pseudintermedius, four low-susceptible original strains (S7-S10; MIC: $1 \mu \mathrm{g} / \mathrm{ml}$ ) and four mutants (SM6SM10) harboured one point mutation (i.e. Ser-80 $\rightarrow$ Ile) in $g r l A$. Of these mutants, three high-level resistant mutants (strains SM7, SM8, and SM10) harboured an additional mutation (i.e. Ser84 $\rightarrow$ Trp or Leu) in gyrA.

Some original strains (i.e. E10, S2 and S5) gave atypical mutants without significant increases in MICs and an additional QRDR mutation emerged after drug exposure, indicating that these original strains have lower incidence of mutations.

\section{MPCs and MPC/MIC ratios}

The MPCs and MPC/MIC ratios for the original strains of $E$. coli were $0.5-32 \mu \mathrm{g} / \mathrm{ml}$ and $4-32$, respectively. In this species, low-susceptible strains with one QRDR mutation (strains E7-E10) had relatively higher MPCs $(8-32 \mu \mathrm{g} / \mathrm{ml})$, compared with high-susceptible strains $(0.5-2 \mu \mathrm{g} / \mathrm{ml})$. P. aeruginosa exhibited similar MPCs $(16-128 \mu \mathrm{g} / \mathrm{ml})$ and MPC/MIC ratios (16-64) for all original strains. In $S$. pseudintermedius, the MPCs $(16-128 \mu \mathrm{g} / \mathrm{ml})$ and MPC/MIC ratios (16-128) in lowsusceptible strains (S7-S10) with one QRDR mutation were higher than those in high-susceptible strains without QRDR mutations (S1-S6; MPC: $2-8 \mu \mathrm{g} / \mathrm{ml}$ and MPC/MIC: 4-16).

According to the published pharmacokinetic data of orbifloxacin, the $\mathrm{C}_{\max }$ and AUC of orbifloxacin at a dose of $7.5 \mathrm{mg} / \mathrm{kg}$ are $6.9 \mathrm{mg} / \mathrm{l}$ and $42.9 \mathrm{mg} . \mathrm{h} / \mathrm{l}$, respectively, were obtained [16]. Using these data, the $C_{\max } / \mathrm{MPC}$ and AUC/MPC ratios were calculated for E. coli, P. aeruginosa, and $S$. pseudintermedius as $0.22-13.8$ $\left(\mathrm{C}_{\max } / \mathrm{MPC}\right)$ and 1.34-85.8 (AUC/MPC), 0.05-0.43 and $0.34-2.68$, and $0.05-3.45$ and $0.34-21.45$, respectively. A comparison among the bacterial species showed that MPC was significantly higher, but the AUC/MPC and $\mathrm{C}_{\max } / \mathrm{MPC}$ ratios were significantly lower, for $P$. aeruginosa compared to E. coli $(P<0.05)$. There were no significant differences in these values between $S$. pseudintermedius and the other two bacterial species. There were also no significant differences in the MPC/MIC ratios among the three bacterial species.

\section{Discussion}

Since introduction of the concept of the MPC, there have been numerous reports of MPCs for fluoroquinolones against Gram-positive and Gram-negative bacteria, but no comparisons of MPCs of fluoroquinolones against different bacterial species under the same experimental conditions. 
Table 1 MICs and MPCs of orbifloxacin and QRDR mutations in the gyrA, parC, and grlA genes of the original stains and mutants used in the study

\begin{tabular}{|c|c|c|c|c|c|c|c|c|}
\hline \multirow{2}{*}{$\begin{array}{l}\text { Parent strains } \\
\text { and mutants }\end{array}$} & \multirow[t]{2}{*}{ MIC $(\mu \mathrm{g} / \mathrm{ml})$} & \multirow[t]{2}{*}{ MIC $(+\mathrm{EPI})^{\mathrm{b}}(\mu \mathrm{g} / \mathrm{ml})$} & \multicolumn{2}{|c|}{ QRDR mutation $^{c}$} & \multirow[t]{2}{*}{ MPC $(\mu \mathrm{g} / \mathrm{ml})$} & \multirow[t]{2}{*}{ MPC /MIC } & \multirow[t]{2}{*}{$\mathrm{C}_{\max } / \mathrm{MPC}^{\mathrm{d}}$} & \multirow[t]{2}{*}{$A \cup C / M P C^{d}$} \\
\hline & & & gyrA & $\operatorname{parC}(\operatorname{gr} \mid A)$ & & & & \\
\hline \multicolumn{9}{|c|}{ E. coli } \\
\hline E1 & 0.063 & $<0.015$ & wt & wt & 1 & 16 & 6.9 & 42.9 \\
\hline E2 & 0.063 & $<0.015$ & wt & wt & 0.5 & 8 & 13.8 & 85.8 \\
\hline E3 & 0.063 & $<0.015$ & wt & wt & 1 & 16 & 6.9 & 42.9 \\
\hline E4 & 0.125 & $<0.015$ & wt & wt & 2 & 16 & 3.45 & 21.45 \\
\hline E5 & 0.125 & $<0.015$ & wt & wt & 2 & 16 & 3.45 & 21.45 \\
\hline E6 & 0.25 & $<0.015$ & wt & wt & 1 & 4 & 6.9 & 42.9 \\
\hline E7 & 0.5 & 0.063 & S83L & wt & 16 & 32 & 0.43 & 2.68 \\
\hline E8 & 1 & 0.063 & D87N & wt & 8 & 8 & 0.86 & 5.36 \\
\hline E9 & 2 & 0.125 & D87N & wt & 8 & 4 & 0.86 & 5.36 \\
\hline E10 & 2 & 0.125 & S83L & wt & 32 & 16 & 0.22 & 1.34 \\
\hline EM1 (0.5) & 1 & 0.125 & S83L & wt & - & - & & \\
\hline EM2 (0.25) & 1 & 0.125 & S83L & wt & - & - & & \\
\hline EM3 (0.5) & 1 & 0.125 & S83L & wt & - & - & & \\
\hline EM4 (1) & 2 & 0.125 & S83L & wt & - & - & & \\
\hline EM5 (1) & 2 & 0.25 & S83L & wt & - & - & & \\
\hline EM6 (0.5) & 2 & 0.125 & S83L & wt & - & - & & \\
\hline EM7 (8) & 4 & 0.125 & S83L & wt & - & - & & \\
\hline EM8 (4) & 4 & 0.25 & D87N & wt & - & - & & \\
\hline EM9 (4) & 8 & 0.25 & D87N & wt & - & - & & \\
\hline EM10 (16) & 4 & 0.125 & S83L & wt & - & - & & \\
\hline \multicolumn{9}{|c|}{ P. aeruginosa } \\
\hline P1 & 1 & 0.015 & wt & wt & 32 & 32 & 0.22 & 1.34 \\
\hline P2 & 1 & 0.031 & wt & wt & 16 & 16 & 0.43 & 2.68 \\
\hline P3 & 1 & 0.063 & wt & wt & 32 & 32 & 0.22 & 1.34 \\
\hline P4 & 2 & 0.125 & wt & wt & 64 & 32 & 0.11 & 0.67 \\
\hline P5 & 2 & 0.063 & wt & wt & 32 & 16 & 0.22 & 1.34 \\
\hline P6 & 2 & 0.125 & wt & wt & 32 & 16 & 0.22 & 1.34 \\
\hline P7 & 2 & 0.031 & wt & wt & 64 & 16 & 0.11 & 0.67 \\
\hline P8 & 2 & 0.063 & wt & wt & 128 & 64 & 0.05 & 0.34 \\
\hline P9 & 4 & 0.25 & wt & wt & 64 & 16 & 0.11 & 0.67 \\
\hline P10 & 4 & 0.125 & wt & wt & 64 & 16 & 0.11 & 0.67 \\
\hline
\end{tabular}


Table 1 MICs and MPCs of orbifloxacin and QRDR mutations in the gyrA, parC, and grlA genes of the original stains and mutants used in the study (Continued)

\begin{tabular}{|c|c|c|c|c|c|c|c|c|}
\hline PM1 (16) & 32 & 0.063 & wt & wt & - & - & & \\
\hline PM2 (8) & 16 & 0.25 & wt & wt & - & - & & \\
\hline PM3 (16) & 16 & 0.5 & wt & wt & - & - & & \\
\hline PM4 (32) & 32 & 1 & T831 & wt & - & - & & \\
\hline PM5 (16) & 32 & 0.25 & wt & wt & - & - & & \\
\hline PM6 (16) & 64 & 0.125 & wt & wt & - & - & & \\
\hline PM7 (32) & 64 & 1 & T831 & wt & - & - & & \\
\hline PM8 (64) & 64 & 0.125 & T831 & wt & - & - & & \\
\hline PM9 (32) & 128 & 2 & T831 & wt & - & - & & \\
\hline PM10 (32) & 16 & 0.25 & wt & wt & - & - & & \\
\hline \multicolumn{9}{|c|}{ S. pseudintermedius } \\
\hline S1 & 0.25 & 0.25 & wt & wt & 2 & 8 & 3.45 & 21.45 \\
\hline$\$ 2$ & 0.25 & 0.5 & wt & wt & 2 & 4 & 3.45 & 21.45 \\
\hline S3 & 0.25 & 0.5 & wt & wt & 8 & 16 & 0.86 & 5.36 \\
\hline S4 & 0.5 & 0.5 & wt & wt & 2 & 8 & 3.45 & 21.45 \\
\hline S5 & 0.5 & 0.5 & wt & wt & 4 & 8 & 1.73 & 10.73 \\
\hline S6 & 0.5 & 0.5 & wt & wt & 4 & 4 & 1.73 & 10.73 \\
\hline S7 & 1 & 1 & wt & S80I & 128 & 128 & 0.05 & 0.34 \\
\hline S8 & 1 & 1 & wt & S801 & 128 & 128 & 0.05 & 0.34 \\
\hline S9 & 1 & 1 & wt & S80I & 64 & 64 & 0.11 & 0.67 \\
\hline S10 & 1 & 1 & wt & S801 & 16 & 16 & 0.43 & 2.68 \\
\hline SM1 (1) & 1 & 1 & wt & wt & - & - & & \\
\hline SM2 (1) & 0.5 & 0.5 & wt & wt & - & - & & \\
\hline SM3 (4) & 1 & 1 & wt & wt & - & - & & \\
\hline SM4 (1) & 2 & 1 & wt & wt & - & - & & \\
\hline SM5 (2) & 0.5 & 0.5 & wt & wt & - & - & & \\
\hline SM6 (2) & 1 & 2 & wt & S80I & - & - & & \\
\hline SM7 (64) & 64 & 128 & S84W & S801 & - & - & & \\
\hline SM8 (64) & 64 & 128 & $\mathrm{~S} 84 \mathrm{~L}$ & S801 & - & - & & \\
\hline SM9 (32) & 8 & 8 & wt & S80I & - & - & & \\
\hline SM10 (8) & 32 & 32 & S84L & S80I & - & - & & \\
\hline
\end{tabular}

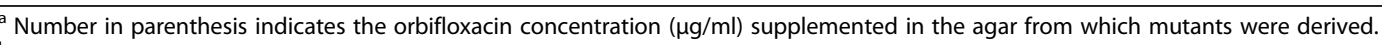

${ }^{\mathrm{b}}$ Minimum inhibitory concentrations (MICs) in the presence of efflux pump inhibitors (EPIs).

c parC of E. coli and P. aeruginosa, and grlA of S. pseudintermedius; wt, wild type; S83L, Ser-83 $\rightarrow$ Leu; D87N, Asp-87 $\rightarrow$ Asn; T83I, Thr-83 $\rightarrow$ Ile; S80I, Ser-80 $\rightarrow$ Ile; S84W, Ser84 $\rightarrow$ Trp; S84L, Ser84 $\rightarrow$ Leu.

Data for maximum concentration ( $\left.C_{\text {max }}: 6.9 \mathrm{mg} / \mathrm{l}\right)$ and area under the concentration time-curve (AUC: $42.9 \mathrm{mg} . \mathrm{h} / \mathrm{l}$ ) of orbifloxacin (dose of $7.5 \mathrm{mg} / \mathrm{kg}$ ) are from reference [16]. 
Thus, this is the first comparison of the MPCs of fluoroquinolones against canine pathogens, and the first determination of the MPC of orbifloxacin against $P$. aeruginosa.

Our results showed that the MPC of orbifloxacin against $P$. aeruginosa is higher than that against E. coli. Pasquali et al. [11] also found that the MPCs of enrofloxacin and ciprofloxacin are higher against $P$. aeruginosa than against E. coli. Collectively, these results indicate that $P$. aeruginosa has a tendency to exhibit higher MPCs for various drugs compared with E. coli. In contrast, the orbifloxacin MPCs against S. pseudintermedius did not differ significantly from those of E. coli and P. aeruginosa. This result may be explained by the considerable variation in MPCs among the strains of S. pseudintermedius. Awji et al. [7] also found that $S$. pseudintermedius exhibited a wider range of orbifloxacin MPCs, compared with those for other veterinary fluoroquinolones. Therefore, the variable MPCs among $S$. pseudintermedius strains are likely to be due to the type of bacterial species and the susceptibility of the pathogen to orbifloxacin.

To examine the basis for the differences in MPCs of orbifloxacin among the three bacterial species, we determined the MICs and fluoroquinolone-resistance mechanisms in MPC mutants of each species. In this study, high-susceptible strains of $E$. coli lacking a QRDR mutation and low-susceptible strains with one QRDR mutation were used as original strains. The MICs of orbifloxacin against all mutants were categorised as susceptible or intermediate based on the CLSI breakpoint criteria for orbifloxacin (MIC $\geq 8 \mu \mathrm{g} / \mathrm{ml}$ ) [12], except for one strain (strain E9), which exhibited a MIC of $8 \mu \mathrm{g} / \mathrm{ml}$. Sequence analysis revealed that all $E$. coli mutants harboured only one QRDR mutation in the $\operatorname{gyr} A$ gene, as also found by Gebru et al. [5]. The two types of gyrA mutations found in the current study (S83L and D87N) are known to cause elevated fluoroquinolone MICs in E. coli $[17,18]$. Generally, MICs of fluoroquinolone against $E$. coli increase in correspondence to the number of QRDR mutations [19], which is the primary mechanism for fluoroquinolone resistance [20]. The emergence of only one QRDR mutation in E. coli in this study may be mainly responsible for the failure to acquire orbifloxacin resistance. Similarly, several studies have shown that most $E$. coli mutants from MPC plates had one or none of QRDR mutations even when parent strains with one gyrA mutation were used [5,6,11]. These findings imply that $E$. coli rarely acquires two or more QRDR mutations in MPC experiments performed under static conditions. In contrast, all strains of $P$. aeruginosa exhibited higher orbifloxacin MICs than the CLSI breakpoint and the MICs were significantly higher than those against E. coli. However, a T83I mutation, which elevates fluoroquinolone MICs $[1,13]$, was detected in fewer mutants of $P$. aeruginosa, compared with $E$. coli, and there were no differences in MICs between $P$. aeruginosa mutants with and without QRDR mutation. These findings suggest that QRDR mutations in $P$. aeruginosa play an insignificant role in the increased MICs of orbifloxacin against the mutants and increased MPC of orbifloxacin.

MICs of orbifloxacin for $P$. aeruginosa and $E$. coli mutants were significantly decreased by addition of an EPI $(\mathrm{PA} \beta \mathrm{N})$ but the effect of this EPI was greater on $P$. aeruginosa strains than on $E$. coli strains. Pasquali and Manfreda [11] similarly found that the decreases in the MICs of enrofloxacin and ciprofloxacin in the presence of PA $\beta \mathrm{N}$ were more pronounced for $P$. aeruginosa than for E. coli. We previously showed that efflux pumps, rather than QRDR mutations, play an important role in the development of fluoroquinolone resistance in $P$. aeruginosa [1]. Differential expression of efflux pumps in $E$. coli and $P$. aeruginosa is likely to be the main factor in the variable increases in orbifloxacin MIC values against mutants of $E$. coli and P. aeruginosa and in orbifloxacin MPCs against these two species.

In S. pseudintermedius, unlike E. coli and P. aeruginosa, orbifloxacin MICs against the mutants and MPCs against the original strains differed markedly based on the susceptibility of the original strain. Sequence analysis revealed three types of QRDR mutations at codon 80 of $\operatorname{grl} A$ and codon 84 of gyrA, which are hotspots for mutations that decrease fluoroquinolone susceptibility in Staphylococcus spp., including S. pseudintermedius [3,8]. High-susceptible original strains lacking the QRDR mutation yielded relatively low orbifloxacin MICs for mutants, which resulted in relatively low MPCs for original strains. In contrast, low-susceptible strains with one QRDR mutation mostly exhibited an additional QRDR mutation after drug exposure and yielded relatively high MICs for mutants, which resulted in relatively high MPCs for original strains. For S. pseudintermedius, the relationship between fluoroquinolone susceptibility of the original strain and the MPC value of orbifloxacin has not been investigated previously. Our results imply that fluoroquinolone susceptibility and the status of QRDR mutations in the original strains can greatly affect the MICs of orbifloxacin against mutants and MPC values for original strains. Further studies are needed to explore these findings. Addition of an EPI did not significantly affect the MICs of orbifloxacin against $S$. pseudintermedius mutants, consistent with the results of Awji et al. [7]. These findings suggest that efflux pumps are not responsible for conferring fluoroquinolone resistance in S. pseudintermedius.

Conversion of in vitro MPCs into clinically useful data requires use of pharmacokinetic and pharmacodynamic parameters of a drug. The $\mathrm{C}_{\max } / \mathrm{MPC}$ and AUC/MPC ratios are important predictors for prevention of the emergence of resistant bacteria. In this study, we obtained these 
parameters based on published $\mathrm{C}_{\max }$ and AUC values for orbifloxacin in dogs [16] and found that both $\mathrm{C}_{\max } / \mathrm{MPC}$ and AUC/MPC for orbifloxacin were lower in $P$. aeruginosa than in $E$. coli, although the values in these two species did not differ significantly from those of S. pseudintermedius. Olofsson et al. [21] suggested that an AUC/MPC ratio $\geq 22$ is predictive of prevention of emergence of a fluoroquinolone-resistant mutant. Thus, our data may imply that appropriate orbifloxacin AUC/MPC ratios cannot be achieved, especially in lowsusceptible strains of E. coli and S. pseudintermedius, and in $P$. aeruginosa strains. However, the orbifloxacin concentration may be higher at infection sites of these bacteria (i.e. urine and skin) than in serum $[22,23]$. Thus, determination of the in vivo AUC/MPC ratio at each infection site is required to evaluate the practical likelihood of the emergence of fluoroquinolone-resistant mutants.

\section{Conclusions}

In conclusion, the results of this study showed that the MPCs and MPC/MIC ratios of orbifloxacin against E. coli, $P$. aeruginosa, and S. pseudintermedius are mainly determined by the primary resistance mechanism of each bacterial species. Notably, E. coli and P. aeruginosa, which are representative Gram-negative bacteria frequently encountered in companion animal medicine, yielded markedly different MPCs of orbifloxacin. MPCs were also affected by the susceptibility (high or low) of the original isolate, especially in S. pseudintermedius. Therefore, the type of bacterial species and the fluoroquinolone susceptibility of the pathogen should be taken into consideration when using fluoroquinolone drugs such as orbifloxacin in canines.

\section{Competing interests}

The authors declare no potential conflicts of interest with respect to the research, authorship, and publication of this article.

\section{Authors' contributions}

$\mathrm{KH}$ designed and TS carried out all the experiments. TS and $\mathrm{KH}$ contributed equally to this study. TS, KH, and YK were involved in preparation of the manuscript. KH drafted the manuscript. All authors read and approved the final version of the manuscript.

Received: 3 December 2012 Accepted: 10 February 2013

Published: 1 May 2013

\section{References}

1. Harada K, Arima S, Niina A, Kataoka Y, Takahashi T: Characterization of Pseudomonas aeruginosa isolates from dogs and cats in Japan: current status of antimicrobial resistance and prevailing resistance mechanisms. Microbiol Immunol 2012, 56:123-127.

2. Harada K, Niina A, Nakai Y, Kataoka Y, Takahashi T: Prevalence of antimicrobial resistance in relation to virulence genes and phylogenetic origins among urogenital Escherichia coli isolates from dogs and cats in Japan. Am J Vet Res 2012, 73:409-417.

3. Onuma K, Tanabe T, Sato H: Antimicrobial resistance of Staphylococcus pseudintermedius isolates from healthy dogs and dogs affected with pyoderma in Japan. Vet Dermatol 2012, 23:17-e5.

4. Blondeau JM: New concepts in antimicrobial susceptibility testing: the mutant prevention concentration and mutant selection window approach. Vet Dermatol 2009, 20:383-396.
5. Gebru E, Choi MJ, Lee SJ, Damte D, Park SC: Mutant-prevention concentration and mechanism of resistance in clinical isolates and enrofloxacin/marbofloxacin-selected mutants of Escherichia coli of canine origin. J Med Microbiol 2011, 60:1512-1522.

6. Gebru E, Damte D, Choi MJ, Lee SJ, Kim YH, Park SC: Mutant prevention concentration and phenotypic and molecular basis of fluoroquinolone resistance in clinical isolates and in vitro-selected mutants of Escherichia coli from dogs. Vet Microbiol 2012, 154:384-394.

7. Awji EG, Tassew DD, Lee JS, Lee SJ, Choi MJ, Reza MA, Rhee MH, Kim TH, Park SC: Comparative mutant prevention concentration and mechanism of resistance to veterinary fluoroquinolones in Staphylococcus pseudintermedius. Vet Dermatol 2012, 23:376-380.

8. Piddock LJ: Mechanisms of fluoroquinolone resistance: an update 1994-1998. Drugs 1999, 58(Suppl 2):11-18.

9. Laboratory NVA: Ministry of Agriculture, Forestry and Fisheries: Sales Amounts and Sales Volumes (Active Substance) of Antibiotics, Synthetic Antibacterials. Tokyo, Japan: Antihelmintics and Antiprotozoals; 2011.

10. Sasaki T, Tsubakishita S, Tanaka Y, Sakusabe A, Ohtsuka M, Hirotaki S, Kawakami T, Fukata T, Hiramatsu K: Multiplex-PCR method for species identification of coagulase-positive staphylococci. J Clin Microbiol 2010, 48:765-769.

11. Pasquali F, Manfreda G: Mutant prevention concentration of ciprofloxacin and enrofloxacin against Escherichia coli, Salmonella Typhimurium and Pseudomonas aeruginosa. Vet Microbiol 2007, 119:304-310.

12. Clinical and Laboratory Standards Institute: Performance Standards for Antimicrobial Disk and Dilution Susceptibility Tests for Bacteria Isolated From Animals; Approved Standard - Third Edition. PA: CLSI document M31-A3 Wayne; 2008.

13. Akasaka T, Tanaka M, Yamaguchi A, Sato K: Type II topoisomerase mutations in fluoroquinolone-resistant clinical strains of Pseudomonas aeruginosa isolated in 1998 and 1999: role of target enzyme in mechanism of fluoroquinolone resistance. Antimicrob Agents Chemother 2001, 45:2263-2268

14. Lee YJ, Cho JK, Kim KS, Tak RB, Kim AR, Kim JW, Im SK, Kim BH: Fluoroquinolone resistance and gyrA and parC mutations of Escherichia coli isolated from chicken. J Microbiol 2005, 43:391-397.

15. Intorre L, Vanni M, Di Bello D, Pretti C, Meucci V, Tognetti R, Soldani G Cardini $G$, Jousson $O$ : Antimicrobial susceptibility and mechanism of resistance to fluoroquinolones in Staphylococcus intermedius and Staphylococcus schleiferi. J Vet Pharmacol Ther 2007, 30:464-469.

16. Walker RD: The use of fluoroquinolones for companion animal antimicrobial therapy. Aust Vet J 2000, 78:84-89.

17. Yoshida H, Bogaki M, Nakamura M, Nakamura S: Quinolone resistancedetermining region in the DNA gyrase gyrA gene of Escherichia coli. Antimicrob Agents Chemother 1990, 34:1271-1272.

18. Oram M, Fisher LM: 4-Quinolone resistance mutations in the DNA gyrase of Escherichia coli clinical isolates identified by using the polymerase chain reaction. Antimicrob Agents Chemother 1991, 35:387-389.

19. Webber M, Piddock $\amalg$ : Quinolone resistance in Escherichia coli. Vet Res 2001, 32:275-284.

20. Chang TM, Lu PL, Li HH, Chang CY, Chen TC, Chang LL: Characterization of fluoroquinolone resistance mechanisms and their correlation with the degree of resistance to clinically used fluoroquinolones among Escherichia coli isolates. J Chemother 2007, 19:488-494.

21. Olofsson SK, Marcusson LL: Komp Lindgren P, Hughes D, Cars O: Selection of ciprofloxacin resistance in Escherichia coli in an in vitro kinetic model; relation between drug exposure and mutant prevention concentration. J Antimicrob Chemother 2006, 57:1116-1121.

22. Matsumoto S, Takahashi M, Yoshida M, Komatsu T, Kitadai Y, Horii Y, Katae H: Absorption, distribution and excretion of orbifloxacin in dogs and cats. J Jap Vet Med Assoc 1997, 50:470-474. in Japanese with English summary.

23. Kay-Mugford PA, Weingarten AJ, Ngoh M, Zolynas R, White A, Katz T, Simmons R, Varma KJ: Determination of plasma and skin concentrations of orbifloxacin in dogs with clinically normal skin and dogs with pyoderma. Vet Ther 2002, 3:402-408.

doi:10.1186/1751-0147-55-37

Cite this article as: Shimizu et al:: Mutant prevention concentration of orbifloxacin: comparison between Escherichia coli, Pseudomonas aeruginosa, and Staphylococcus pseudintermedius of canine origin. Acta Veterinaria Scandinavica 2013 55:37. 